\title{
实轴上 $C^{1}$ 微分同胚的 $C^{1}$ 共轭分类*
}

\author{
章 梅 荣 \\ (清华大学应用数学系, 北京 100084)
}

\section{关镜词 光滑共轭、光滑线性化、能入流}

本文将对一类 $\mathrm{R}$ 上的局部的和整体的 $C^{1}$ 微分同胚给出其在 $C^{1}$ 共轭下的完全分类. 对 $r-1,2, \cdots, \infty, \infty$, 记 $D^{r}(0)-\left\{f: \mathrm{R} \rightarrow \mathrm{B}\right.$ 是 $C^{r}$ 的, $f$ 以 0 为唯一的不动点, 又 $\left.f^{\prime}(x)>0, x \in \mathbf{R}\right\}$. 文献 $[1,2]$ 系统地讨论了 $f \in D^{r}(0)$ 的光滑嵌人流的存在性以及其它相关 的问题，证明了以下分类问题仅有数值不变量：（i） $f \in D^{r}(0)(r \geqslant 2)$ 且 $\lambda-f^{\prime}(0)>0$, $\neq 1$ 在 $C^{s}(1 \leqslant s \leqslant r)$ 共轭下的分类; (ii) 部分的 $f \in D^{r}(0)(r \geqslant 2)$ 且 $\lambda=1$ 在 $C^{1}$ 共轮 下的分类. 文献 $[3,4]$ 证实了对 $N D^{\infty}(0)-\left\{f: f(z)-z+a z^{2}+\cdots\right.$ 在 $z=0$ 附近解析 $\}$ $(a \neq 0)$ 在解析共轭下的分类会出现函数不变量. 对高维系统在 $C^{\infty}$ 共轭下的分类工作可以 参见文献 [5]. 本文证明了对 $\mathbf{R}$ 上的 $C^{1}$ 微分同肧的局部 $C^{1}$ 共轭分类也会出现函数不变量.

\section{1 函数不变量的构造}

本文将采用嵌入流的方法来讨论. 对微分同胚的 $C^{s}$ 共轭, $C^{s}$ 线性化与 $C^{s}$ 嵌入流的 概念,参见文献 $[3,6,2]$.

设 $f \in D^{\prime}(0)$ 且 $\lambda=f^{\prime}(0)>0$, $\neq 1$, 记 $\sigma=-(+)$ 如果 $\lambda<1(\lambda>1)$, 则

$$
\lim _{n \rightarrow \infty} f^{n}(x)=0 \text {. }
$$

对 $c>0$, 记

$$
\left(p_{n}^{\prime}(x, c)-\right) p_{n}(x, c)-\frac{\left(f^{n}\right)^{\prime}(x)}{\left(f^{n}\right)^{\prime}(c)} .
$$

记 $E^{+}(0)=\left\{f \in D^{1}(0): \lambda \neq 1\right.$ 且 $f$ 在 $\mathbf{R}^{+}-[0, \infty)$ 上有一个 $C^{1}$ 的人流 $\}$.

命题 $1^{[2]}$ (i) 设 $f \in D^{1}(0)$ 且 $\lambda \neq 1$. 则 $f$ 在 $R^{+}$上有 $C^{1}$ 嵌人流 $\Leftrightarrow$ 存在 $c>0$ (因而对一切 $c>0$ ) 有 $\lim _{n \rightarrow \infty} p_{n}(x, c)$ 在 $x \in(0, \infty)$ 的任一紧子集上是一致收敛的. (ii) 若 $f \in E^{+}(0)$, 则 $f$ 在 $\mathrm{R}^{+}$上的 $C^{1}$ 族入流是唯一的. (iii) 若 $f^{\prime}(x)$ 在 $(0, \infty)$ 的任一紧子集 上有有界变差, 则 (i) 的收玫条件满足. 因此若 $f \in D^{r}(0)(r \geqslant 2)$, 无论 $\lambda \neq 1, \lambda-1, f$ 在 $\mathbf{R}^{+}$上均有唯一的 $C^{1}$ 烷人流.

设 $f \in E^{+}(0)$ 及 $c>0$, 利用文献 [2]中的嵌人向量场, 不难验证下述序列

$$
S_{c}(x)=\lim _{n \rightarrow \infty \infty} \frac{f^{n}(x)}{f^{n}(c)}, x \in \mathbb{R}^{+},
$$

1991-10-19 收稿, 1992-12-24 收修改稿.

* 清华大学理学院基金资趿课题. 
在 $x \in(0, \infty)$ 的任一紧子集上是一致收玫的. 于是 $f$ 在 $\mathrm{R}^{+}$上的 $C^{1}$ 嵌人流 $\left\{f^{t}\right\}(t \in \mathrm{R})$ 可 以表示成

$$
f^{t}(x)-S_{c}^{-1}\left(\lambda^{t} S_{c}(x)\right), x \in \mathbf{R}^{+}, t \in \mathbf{R} .
$$

另外, 上述 Schröder 函数 $\left\{S_{c}: c>0\right\}$ 还有以下性质:

(S1) $S_{c}: \mathrm{R}^{+} \rightarrow \mathrm{R}^{+}$是个同胚且 $S_{c}(0)=0$;

(S2) 对 $x>0, S_{c}^{\prime}(x)-\frac{\mathrm{d}}{\mathrm{d} x} S_{c}(x)$ 存在且大于 0 ;

(S3) $S_{d}(x) \equiv S_{c}(x) / S_{c}(d), c, d>0$.

一般情况下, $S_{c}^{\prime}(0)$ 不存在. 对 $c>0$, 令

$$
\left(\phi_{c}^{\prime}(x)-\right) \phi_{c}(x)=S_{c}^{\prime}\left(S_{c}^{-1}(x)\right), x>0 .
$$

这些将作为 $C^{1}$ 共轭的函数不变量.

引进在一点处 (如 $x-0$ ) 的渐近齐次函数的空间如下: $A H^{+}(0)-\{\phi:(0, \infty) \rightarrow \mathrm{R}$ 是 连续的正函数,且 $(H 1)$ 积分 $\int_{0}^{1} \frac{\mathrm{d} x}{\phi(x)}$ 收玫; $(H 2) \frac{x}{p(x)} \rightarrow 0$ 当 $x \rightarrow 0^{+}$;

$$
\text { (H3) } \left.\lim _{(P, x) \rightarrow\left(q, 0^{+}\right)} \frac{\phi(p x)}{\phi(x)}-1, \forall q>0\right\} \text {. }
$$

在 $A H^{+}(0)$ 上定义等价关系 为: $\phi \sim \phi \Leftrightarrow \lim _{x \rightarrow 0^{+}} \frac{\phi(x)}{\phi(x)}$ 有正极限. 例如, 正常数函数在 $A H^{+}(0)$ 中且相互等价; 又如 $\phi_{\mu}(x)-(-\ln x)^{\mu}, 0<x<1$, 对 $\forall \mu \in \mathrm{R}$ 可认为在 $A H^{+}(0$ 中且 $\phi_{\mu} \phi_{\nu}$ 若 $\mu \neq \nu . A H^{-}(0), A H^{+}(a), A H^{-}(a)$ 以及相应的等价关系也可以类似 定义.

命题 2 设 $f \in E^{+}(0)$, 则 $\phi_{c}^{t} \in A H^{+}(0)$ 且 $\phi_{c}^{\prime} \sim \phi_{d}^{f}, \forall c, d>0$.

证 对 $c>0$, 由(3)式知

$$
\int_{x}^{1} \frac{\mathrm{d} t}{\phi_{c}(t)}-\int_{x}^{1} \frac{\mathrm{d} t}{S_{c}^{\prime}\left(S_{c}^{-1}(t)\right)}-S_{c}^{-1}(1)-S_{c}^{-1}(x)
$$

收敛到 $S_{c}^{-1}(1)$, 当 $x \rightarrow 0^{+}$. 对 $(H 2)$, 由于

$$
\frac{\partial}{\partial t} f^{t}(x)=\frac{\lambda^{t} S_{c}(x) \ln \lambda}{S_{c}^{\prime}\left(S_{c}^{-1}\left(\lambda^{t} S_{c}(x)\right)\right)}-\frac{y}{\phi_{c}(y)},\left(y-\lambda^{t} S_{c}(x)\right)
$$

以及

$$
\lim _{(t, x) \rightarrow\left(s, y^{+}\right)} \frac{\partial}{\partial t} f^{t}(x)-\left.\frac{\partial}{\partial t} f^{\prime}(x)\right|_{(s, 0)}-0
$$

因此 $(H 2)$ 满足. 同理，由于

$$
\frac{\partial}{\partial x} f^{\prime}(x)=\frac{\lambda^{t} S_{c}^{\prime}(x)}{S_{c}^{\prime}\left(S_{c}^{-1}\left(\lambda^{t} S_{c}(x)\right)\right)}-\frac{\lambda^{t} \phi_{c}(y)}{\phi_{c}\left(\lambda^{t} y\right)},\left(y-S_{c}(x)\right)
$$

以及

$$
\lim _{(t, x) \rightarrow\left(s, 0^{+}\right)} \frac{\partial}{\partial x} f^{t}(x)-\left.\frac{\partial}{\partial x} f^{t}(x)\right|_{(s, 0)}-\lambda^{\prime},
$$

因此 $(H 3)$ 也满足. 故 $\phi_{c} \in A H^{+}(0)$. 至于 $\phi_{c} \sim \phi_{d}$ 可以从 $(S 3)$ 直接验证. 


\section{2 分类 结果}

定理 1 (i) $f \in E^{+}(0)$ 对应于 $A H^{+}(0)$ 中的一个等价类 $\left[\phi^{f}\right]$. 反之,对任意 $\lambda>0$, $\neq 1$ 及 $\phi \in A H^{+}(0)$, 必存在 $f \in E^{+}(0)$ 使得 $f^{\prime}(0)-\lambda$ 且 $\phi^{f} \sim \phi$. (ii) $f, g \in E^{+}(0)$ 是 $C^{1}$ 共轭的 $\Leftrightarrow f^{\prime}(0)=g^{\prime}(0)$ 且 $\phi^{t} \sim \phi^{8}$.

证 (i) 仅需证后一结论。设 $\lambda>0, \neq 1$ 与 $\phi \in A H^{+}(0)$ 给定. 由于 $\phi$ 满足 $(H 1)$, 可令

$$
B(x)=\int_{0}^{x} \frac{\mathrm{d} t}{p(t)}, \quad x \in \mathrm{R}^{+} .
$$

于是 $B$ 满足 (S1), (S2), 因此可定义出 $\mathbf{R}^{+}$上的一个连续流

$$
f^{t}(\boldsymbol{x})=B\left(\lambda^{t} B^{-1}(\boldsymbol{x})\right), x \in \mathbf{R}^{+}, t \in \mathbf{R} .
$$

由 $\phi \in A H^{+}(0)$ 的性质, 不难验证(4)式定义出 $\mathbf{R}^{+}$上的一个 $C^{1}$ 流. 令 $f-f^{1}$, 则 $f^{\prime}(0)-$ 2 , 且 $f \in E^{+}(0)$. 进一步, 由(1),(4)式可得: 对 $c, x>0$,

$$
\begin{aligned}
S_{c}(x) & =\lim _{n \rightarrow \infty} \frac{B\left(\lambda^{n} B^{-1}(x)\right)}{B\left(\lambda^{n} B^{-1}(c)\right)}-\lim _{t \rightarrow \infty} \frac{B\left(\lambda^{t} B^{-1}(x)\right)}{B\left(\lambda^{t} B^{-1}(c)\right)} \\
& =\lim _{t} \frac{B^{\prime}\left(\lambda^{t} B^{-1}(x)\right)}{B^{\prime}\left(\lambda^{t} B^{-1}(c)\right)} \frac{B^{-1}(x)}{B^{-1}(c)}=\lim _{t} \frac{\phi\left(\lambda^{t} B^{-1}(c)\right)}{\phi\left(\lambda^{t} B^{-1}(x)\right)} \frac{B^{-1}(x)}{B^{-1}(c)} \\
& =\frac{B^{-1}(x)}{B^{-1}(c)},
\end{aligned}
$$

因此可得: $\phi_{c}^{t}(x)=S_{c}^{\prime}\left(S_{c}^{-1}(x)\right)=\frac{\phi\left(x B^{-1}(c)\right)}{B^{-1}(c)}$, 故又有 $\phi_{c}^{f} \sim \phi$.

(ii) 必要性: 设 $f, g \in E^{+}(0)$ 且存在一个 $C^{1}$ 微分同胚 $h: \mathbf{R}^{+} \rightarrow \mathbf{R}^{+}$使得 $h(f(x)) \equiv$ $g(h(x)), x \in \mathbf{R}^{+}$. 自然 $h(0)=0$, 这等式在 $x=0$ 点的导数表明 $f^{\prime}(0)-g^{\prime}(0) \stackrel{\text { 记 }}{=} \lambda$, 设 $\left\{f^{t}\right\},\left\{g^{t}\right\}$ 是 $f, g$ 的 $C^{1}$ 蛀人流, 由嵌人流的唯一性, 我们有

$$
h\left(f^{t}(x)\right) \equiv g^{t}(h(x)), x \in \mathbf{R}^{+} .
$$

对 $c>0$ 令 $d-h(c)$ 及 $H=S_{d}^{g} \circ h \circ\left(S_{c}^{\prime}\right)^{-1}$, 则 $H: \mathbf{R}^{+} \rightarrow \mathbf{R}^{+}$是同肧且在 $(0, \infty)$ 上可 导. 利用貯人流的表达式(2)及等式(5)可知 $H\left(\lambda^{t} x\right) \equiv \lambda^{t} H(x), x \in \mathbf{R}^{+}, t \in \mathbf{R}$. 对 $x$ 求导说明 $H^{\prime}(x)(x>0)$ 是正常数函数, 故 $H(x) \equiv \gamma x, x \in \mathbf{R}^{+}$, 其中 $\gamma>0$. 记 $\tau--\ln \gamma / \ln \lambda \in \mathbf{R}$ 及 $G-g^{\mathrm{r}} \circ h$, 是 $G: \mathbf{R}^{+} \rightarrow \mathbf{R}^{+}$是 $C^{1}$ 微分同胚, 因此有

$$
\lim _{x \rightarrow 0^{+}} \frac{\phi_{c}^{\prime}(x)}{\phi_{c}^{\prime}(x)}=\lim _{x \rightarrow 0^{+}} G^{\prime}\left(\left(S_{c}^{t}\right)^{-1}(x)\right)=G^{\prime}(0)>0,
$$

故 $\phi_{c}^{!} \sim \phi_{\dot{q}}^{q}$.

(iii) 充分性: 设 $f^{\prime}(0)-g^{\prime}(0)-\lambda$ 且有 $c, d, \gamma>0$ 使得

$$
\lim _{x \rightarrow 0^{+}} \frac{\phi_{c}^{\prime}(x)}{\phi_{d}^{\prime}(x)}-r,
$$

则令 $h=\left(s_{d}^{g}\right)^{-1} \circ S_{c}^{\prime}, x \in \mathbf{R}^{+}$, 可以验证 $h^{\prime}(0)-\gamma$ 存在且 $h$ 是 $\mathbf{R}^{+}$上的 $C^{1}$ 微分同胚, 又 $h^{\circ} f=g \circ h$ 在 $\mathbf{R}^{+}$上成立.

为了考虑 $E(0)-\left\{f \in D^{1}(0): \lambda \neq 1\right.$ 且 $f$ 在 $\mathrm{R}$ 上有 $C^{1}$ 说入流 $\} E^{+}(0) \cap E^{-}(0)$ 的 $C^{1}$ 共轮分类, 可以以 $\phi_{0}^{t} \in A H^{o}(0)(\sigma-+,-)$ 作为函数不变量. 但因为共轮变换可能是反 
定向的, 故在 $A H^{+}(0) \times A H^{-}(0)$ 上定义等价关系 为: $\left(\phi_{+}, \phi_{-}\right) \sim\left(\psi_{+}, \phi_{-}\right) \Leftrightarrow$ 下述之一 满足:

(i) $\phi_{\sigma}(x) / \phi_{\sigma}(x)$ 有正极限, 当 $x \rightarrow 0 \sigma, \sigma=+,-$;

(ii) $\phi_{\sigma}(x) / \phi_{-\sigma}(x)$ 有正极限, 当 $x \rightarrow 0 \sigma, \sigma=+,-$.

定理 2 (i) 设 $f, g \in E(0)$. 则 $f, g$ 是 $C^{1}$ 共轭 $\Leftrightarrow f^{\prime}(0)=g^{\prime}(0)$ 且在 $A H^{+}(0) \times$ $A H^{-}(0)$ 中 $\left(\phi_{+}^{t}, \phi_{-}^{t}\right) \sim\left(\phi_{+}^{f}, \phi_{-}^{\ell}\right)$. (ii) $f \in E(0)$ 在 $x=0$ 附似可以 $C^{1}$ 线性化 $\Leftrightarrow \phi_{0}^{t}$ 是 $A H^{\sigma}(0)$ 意义下的常数函数, $\sigma=+$, -

证 (i) 同定理 1. (ii) 若 $f$ 可以 $C^{1}$ 线性化,则 $S_{c}^{\prime}(c>0)$ 是 $\mathbf{R}^{+}$上的 $C^{1}$ 微分同 肧, 故 $\phi_{c}^{\prime}(x)-S_{c}^{\prime}\left(S_{c}^{-1}(x)\right) \rightarrow S_{c}^{\prime}(0)>0$ 当 $x \rightarrow 0^{+}$. 因此在 $A H^{+}(0)$ 中可以认为是常数函 数.

对 $\mathbf{R}$ 上的整体微分同胚,仅需讨论仅有两个不动点的情形. 设 $I=[a, b] \subset \mathbf{R}$, 记

$$
E(I)=\left\{f \in \operatorname{Diff}^{1}(I): f\right.
$$

仅有不动点 $\left.a, b ; f^{\prime}(x)>0, x \in I ; f \in E^{+}(a) \cap E^{-}(b)\right\}$. 对 $f \in E(I)$, 除本文的 $\phi_{+}^{f} \in A H^{+}(a)$, $\phi_{-}^{t} \in A H^{-}(b)$ 外, 文献[2]中还定义了一个 $C^{1}$ 的以 1 为周期的函数 $\psi^{f}$ 作为“连接” 不动点 之间的整体不变量. $\psi^{t} \sim \psi^{z}$ 意即存在 $t_{0}$ 使得 $\phi^{f}(t) \equiv \psi^{z}\left(t+t_{0}\right), t \in \mathbf{R}$. 故有

定理 3 设 $f, g \in E(I)$. 则 $f, g$ 是保向地 $C^{1}$ 共轭的 $\Leftrightarrow(\mathrm{i}) f^{\prime}(a)-g^{\prime}(a)$ 在 $A H^{+}(a)$ 中 $\phi_{+}^{f} \sim \phi_{+}^{g}$; (ii) $f^{\prime}(b)-g^{\prime}(b)$ 且在 $A H^{-}(b)$ 中 $\phi_{-}^{f} \sim \phi_{-}^{g}$; (iii) $\psi^{f} \sim \psi^{g}$.

下面举一个例子. 考虑下述微分方程

$$
\frac{\mathrm{d} x}{\mathrm{~d} t}=V_{\mu \nu}(x)=\left\{\begin{array}{l}
\lambda\left(1-\frac{\mu}{\ln x}\right) x, 0 \leqslant x<1, \\
\lambda\left(1-\frac{\nu}{\ln |x|}\right) x,-1<x \leqslant 0,
\end{array}\right.
$$

其中 $\lambda \neq 0, \mu, \nu \in \mathrm{R}$. 方程(6)生成一个 $C^{1}$ 流

$$
f_{\mu \nu}^{t}(x)=\left\{\begin{array}{l}
B_{\mu}^{-1}\left(\mathrm{e}^{\lambda t} B_{\mu}(x)\right), 0 \leqslant x \ll 1, t \in \mathbf{R}, \\
B_{\nu}^{-1}\left(\mathrm{e}^{\lambda t} B_{\nu}(x)\right),-1 \ll x \leqslant 0, t \in \mathbf{R},
\end{array}\right.
$$

其中

$$
B_{\alpha}(x)=x(\alpha-\ln |x|)^{\alpha},|x| \ll 1 .
$$

于是 $f_{\mu \nu}-f_{\mu \nu}^{1} \in E(0)$. 容易验证: (i) 当 $x \rightarrow 0^{+}$时 $\phi_{+}^{\prime \mu \nu}(x)-B_{\mu}^{\prime}\left(B_{\mu}^{-1}(x)\right) \sim(-\ln x)^{n}$; (ii) 当 $x \rightarrow 0^{-}$时, $f_{-}^{j \mu \nu}(x) \sim(-\ln |x|)^{\nu}$. 因此 $f_{\mu \nu}$ 是 $C^{1}$ 共轭于 $f_{\alpha \beta} \Leftrightarrow \mu-\alpha, \nu=\beta$ 或 者 $\mu-\beta, \nu-\alpha$.

\section{参考文献}

[1] 章梅荣, Ac ta Math. Sinica, New Series, 1992, (8): 148-157.

[2]章梅荣、李伟固,数学进展, 1992, 21: 245-246.

[3] Anosov, D. V., Arnold, V. I., Dynamical Systems /, Springer-Verlag, Berlin, 1988, 92 -96.

[4] Belitskii, G. R., Siberian J. Math., 1986, 27: 801-804.

[5] Palis, J., Yoccoz, J. C., Bal. Soc. Bras. Mat. (Nova Série), 1990, 20: 25-47.

[6] Lam, P. F., Colloq. Math., 1976, 35: 275-287. 